\title{
La docencia como ciencia productiva en el marco de la responsabilidad social del profesor universitario
}

\section{Teaching science as production under the social responsibility of university professor.}

\author{
Luis José Vera Guadrón \\ Doctor en Ciencias de la Educación Universidad Rafael Belloso Chacín. Profesor y Coordinador línea de Investigación Formación Docente \\ en Universidad Nacional Experimental Rafael María Baralt. luisjverag@hotmail.com \\ Yaneth Coromoto Acosta Bautista \\ Doctora en Ciencias Humanas. Universidad del Zulia. Profesora Titular Universidad Nacional Experimental Rafael María Baralt. \\ acostayaneth@hotmail.com
}

\section{Gladys Contreras}

Doctora en Ciencias Mención Investigación Universidad Rafael Belloso Profesora Universidad Nacional Experimental Rafael María Baralt.

Chacíngtcontreras@hotmail.com

Para citar este artículo:

Vera, C. L., Acosta, B. Y., Contreras, G. (2014). La Docencia Como Ciencia Productiva en el Marco de la Responsabilidad Social Del Profesor Universitario. Escenarios. 12(1), 50-59.

Recibido: octubre 12 de 2013

Aceptado: enero 30 de 2014

\begin{abstract}
RESUMEN
Se analiza la docencia en la educación superior como ciencia productiva en el marco de la responsabilidad social del profesor universitario en el Programa Educación de la Universidad Nacional Experimental Rafael María Baralt, desde los resultados investigativos financiados por el Consejo de Desarrollo Científico, Humanístico y Tecnológico de la mencionada universidad. La metodología fue cuantitativa, el tipo de investigación analítica, con diseño de campo, no experimental y transversal; la población del estudio fue de 410 profesores del Programa Educación, la muestra de 80 sujetos. Para recolectar la información se elaboró un instrumento de 69 itemes con escala tipo Likert, sometido a validez y confiabilidad para su aplicación. Los datos se procesaron mediante la estadística inferencial, con la prueba Tukey y el Anova de un factor. Los resultados obtenidos indican que la docencia en la educación superior como ciencia productiva en el marco de la responsabilidad social del profesor universitario, es considerada como una gestión científica al ser realizarla conscientemente con fundamento en teorías y articularla con la investigación y extensión, cumpliendo con la misión de la universidad; la sistematización de experiencias y proyectos de investigación, tuvieron menor posicionamiento en la práctica mediadora y en investigación respectivamente; recomendándose acciones para promover la docencia como ciencia productiva, pues se genera conocimiento, mediante proyectos de investigación, que pueden apuntar a la productividad, la sistematización y reflexión sobre la teoría y la práctica de la docencia.
\end{abstract}

Palabras clave: docencia universitaria; ciencia productiva; sistematización de experiencias; proyectos de investigación; responsabilidad social. 


\section{ABSTRACT}

Discusses the teaching in higher education as productive science within the framework of the social responsibility of University Professor in the program at the Universidad Nacional Experimental Rafael Maria Baralt, completed and funded by the Council of Scientific, Humanistic and Technological university said. The methodology was quantitative, analytical type of research, field design, experimental and not cross, the study population was 410 Teachers Education Program, the sample of 80 subjects. To collect information itemes an instrument with 69 Likert scale, subject to validity and reliability for application was developed. The data were processed using inferential statistics, with Tukey and a factor Anova test. The results indicate that teaching in higher education as a productive science in the context of the social responsibility of the university professor, is regarded as a scientific management to do it consciously based on theories and articulate research and extension, because it meets the mission college, the systematization of experiences and research projects, with lower positioning mediation practice and research respectively, actions are recommended to promote teaching as a productive science, because knowledge is generated through research projects, which can point to the productivity, the systematization and reflection on the theory and practice of teaching.

Keywords: university teaching, productive science, systematization of experiences, research projects, social responsibility.

\section{INTRODUCCIÓN}

La educación universitaria a nivel mundial ha tenido relevancia por los diferentes estados, los organismos multilaterales y la sociedad al considerarla como la palanca que puede promover el desarrollo socioeconómico de un país, a partir de la formación de profesionales altamente calificados, en la generación de conocimiento científico y tecnológico orientado a dinamizar la economía mediante la explotación de los recursos naturales a fin de transformarlos en bienes y servicios tendentes a satisfacer las necesidades de la población y al bienestar social de las comunidades.

Resulta oportuno, reseñar el planteamiento de la Organización de la Naciones Unidas para el desarrollo de la Ciencia, la Cultura y la Educación (UNESCO. 2009), quien considera a la Educación Superior como un bien público y asigna la responsabilidad a los gobiernos que suscriben el documento, por ello las autoridades universitarias y los profesores como responsables de concretar el currículo, a través de la docencia, la investigación, la extensión y la gestión, deben liderar la sociedad en la generación de conocimiento, para atender los problemas que afectan al bienestar de la población local y global.

Es evidente entonces, que la Universidad tiene como misión generar conocimiento útil para la sociedad, según Vallaeys y Col (2008), mediante la formación de la población, como una de las funciones asignadas por la sociedad, implica su manera de entender e interpretar el mundo, comportarse en él y valorar ciertas cosas en su vida. Influye asimismo, sobre la deontología que orienta la definición de la ética profesional de cada disciplina y su rol social.

Por consiguiente, el proceso de formación de profesionales y de generación de conocimiento, implica develar la responsabilidad social del profesor en la función docente, pues lo compromete a desarrollar un conjunto de estrategias relacionadas con las actividades didácticas para garantizar la gestión del conocimiento en la formación del profesional requerido por la sociedad o entorno, quien tendrá la responsabilidad de participar activamente en la dinamización del proceso productivo, la solución de problemas, con su acción orientada al bienestar social de las generaciones presentes y futuras.

Sobre la base de las ideas expuestas, la función docente como responsabilidad social del profesor universitario, está orientada a promover la participación del estudiante en la construcción del conocimiento, al sistematizar las experiencias de aprendizaje, según Castillo y Cabrerizo (2006), es considerada una actividad científica, al utilizar el método dialógico, el trabajo en equipo, uso de estrategias creativas, vincular el contenido con el contexto, valorar la deontología profesional, reflexionar sobre la praxis docente y la aplicación del conocimiento. Esta acción docente debe quedar registrada para socializarla con sus pares y someterla al proceso de revisión y validación con el fin de crear metodologías y tecnologías creativas en la generación del aprendizaje.

En este sentido, al realizar una evaluación del comportamiento de la docencia universitaria, la mayoría de los estudios consideran imperativo profundizar en contenidos y exigencias para la formación de los profesionales requeridos por la sociedad, pues el método de enseñanza tradicional, ha formado un sinnúmero de profesionales que han hecho posible el avance de la ciencia y la tecnología en los últimos años, pero ha sido superado por las innovaciones educativas, sin embargo, según Vera et al (2012: 270), "existe un alto empleo de estrategias para ejercer la función de docencia y un moderado uso de las actividades vinculadas con la investigación, la extensión y la gestión de procesos productivos".

En este propósito, las diferentes ordenaciones legales, así como los cambios paradigmáticos obligan a reordenar curricularmen- 
te a las instituciones universitarias, para dar respuestas a las funciones básicas relacionadas entre sí, como son la docencia, investigación y extensión. El profesor a tiempo completo y dedicación exclusiva, es el encargado de llevarlas a cabo, sin embargo este es un problema digno de analizar, pues la tendencia de la universidad se hace cada vez mayor hacia la docencia como actividad predominante.

En las universidades venezolanas ha predominado la tendencia orientada a la docencia, de acuerdo con Inciarte et al (2010), el principal objetivo de estas instituciones corresponde a la profesionalización mediante la aplicación de esquemas de trabajo docente basados en la transmisión y reproducción de contenidos, relegando la implementación de políticas de investigación y de extensión, al sobrecargar a los profesores con 16 y más horas de clases académicas, en cuatro cursos de sesenta alumnos cada uno aproximadamente, lo cual limita al profesor su tiempo y dedicación a la investigación y a la extensión.

La problemática planteada en torno a la investigación y a la extensión se observa en la baja proporción de investigación financiada y no financiada y en los proyectos de extensión, los cuales son casi nulos si se aprecia que el $90 \%$ de los profesores tiene dedicación exclusiva y tiempo completo, por ello es importante que las Instituciones de Educación Universitaria, asuman su responsabilidad social con las comunidades internas y las de su entorno, en la generación de conocimiento, ciencia y tecnología.

En efecto, la investigación realizada por Albornoz (2010), sobre los factores que influyen y condicionan la alta y baja tasa de productividad académica en América Latina y el Caribe, logró clasificar los factores como externos, institucionales y personales, del mismo modo examinó la experiencia cuantitativa de las fases secuenciales de una cadena de producción académica, que va desde el consumo hasta el efecto del conocimiento, especialmente referido a los casos de México y Venezuela. Concluyó entre otras cuestiones, que la comunidad académica estudiada, es más consumidora que productora de conocimientos y que los factores institucionales afectan más el rendimiento en esa área que los factores externos y los personales.

Es evidente entonces, que la problemática planteada en la docencia universitaria es resultado de la masificación de las universidades, quienes para atender la demanda, se ha incorporado a un gran número de profesores sin la formación requerida para promover un aprendizaje que le permita al estudiante utilizar el conocimiento para resolver sus problemas académicos, personales, profesionales y del entorno, pues la baja calidad se ve reflejada en una alta proporción de estudiantes aplazados, repitencia estudiantil, baja cualificación para desempeñarse en su campo laboral, provocando entre otras causas, desempleo y marginación de la población, situación preocupante si se considera la inversión que realiza la sociedad.
Con referencia a lo anterior, se hace necesario implementar acciones tendentes a disminuir la repitencia y la deserción, así como elevar el rendimiento en cuanto a la formación del talento humano, es decir la formación de un profesional calificado para desempeñarse con calidad en su área laboral, que se constituya en un emprendedor generador de conocimiento, empleo y de riqueza que contribuya a disminuir los niveles de pobreza y a elevar la producción de bienes y servicios, orientados al bienestar individual y colectivo de la población.

Al respecto, en una investigación realizada por Ruz (2009), cuyo objetivo fue determinar la responsabilidad social de las instituciones públicas de educación superior en el estado Zulia, Venezuela, evidenció que existe una responsabilidad social interna de las universidades del estado Zulia, la cual se mide a través de los impactos de funcionamiento organizacional, manifestado en su personal como miembros activos y agentes multiplicadores de su política de bienestar social, gestionando los recursos humanos con los que cuenta la universidad y una responsabilidad social externa emprendida hacia la comunidad y el empresariado, quienes exigen un perfil profesional de los egresados acordes con las necesidades del sector productivo del entorno de la localidad.

En el estudio realizado por Soto (2008), cuyo propósito fue evaluar el impacto de la responsabilidad social universitaria en la conformación del perfil del egresado de Educación Integral de la Universidad Pedagógica Experimental Libertador, obtuvo como resultado que el impacto entre la responsabilidad social universitaria y el perfil del egresado no está acorde con los lineamientos de la universidad, por ello recomendó fortalecer la gestión universitaria en función de la responsabilidad social.

Como puede observarse, son múltiples los factores que afectan el desarrollo de la docencia universitaria en cuanto a la integración o articulación de las funciones, las cuales deben ser operacionalizadas por los profesores universitarios, se devela la existencia de una primacía de la docencia de aula desvinculada de la investigación y de la extensión como actividades generadoras de conocimiento.

Ante la situación planteada, surge la necesidad de realizar la investigación, cuyo objetivo fue analizar la docencia en la educación superior como ciencia productiva en el marco de la responsabilidad social del profesor universitario en el Programa Educación de la Universidad Nacional Experimental Rafael maría Baralt (UNERMB), de manera que los profesores ofrezcan sus opiniones y las fortalezas que poseen en una determinada disciplina, pues se considera importante indagar sobre la actuación de los profesores en la articulación de las funciones de docencia, investigación, extensión en la actividad mediadora de una cátedra en particular, a fin de garantizar la calidad, donde el estudiante sea partícipe en la construcción de su propio conocimiento. 
Sobre la base de las consideraciones anteriores, el Consejo de desarrollo Científico, Humanístico y Tecnológico (CDCHT) del Programa Investigación de la UNERMB, aprobó financiamiento para realizar la presente investigación, la cual se concluyó en 2012 y el informe se entregó en mayo de 2013; la metodología seleccionada fue el enfoque cuantitativo la población fue de 410 profesores del Programa Educación, la muestra se constituyó por 80 sujetos. Como resultado se obtuvo una media de 4,44 para la docencia en la educación superior como ciencia productiva en el marco de la responsabilidad social del profesor universitario.

\section{La docencia como ciencia productiva en el marco de la responsabilidad del profesor universitario}

La docencia universitaria es una de las funciones del profesor que permite la posibilidad de pensar la educación dirigida a brindarle a cada estudiante las herramientas e instrumentos para desarrollar plenamente sus potencialidades, en cuanto a lo personal y profesional. En ese sentido, la docencia como ciencia productiva es considerada una actividad rigurosa, coherente y predictiva en cuanto esté fundamentada conscientemente en el hecho educativo, donde las ciencias de la educación tienen su objeto y la didáctica, dado que es una ciencia práctica y productiva, coadyuvan al trabajo del profesor el cual debe estar orientado a vincular la enseñanza con el contexto, para establecer relaciones teóricas y prácticas orientadas a la comprensión de la realidad.

En efecto, la función docencia es definida por el Programa de Beneficio Académico de los Profesores Universitarios de Venezuela (2002: 14), “como una función básica de la institución, la cual implica la planificación, desarrollo y evaluación en forma sistemática y sustentada teóricamente de la enseñanza y el aprendizaje", igualmente está referida a otros procesos relacionados con la construcción y difusión del saber en áreas o disciplinas determinadas, que son administradas por los profesores.

Por consiguiente, la docencia universitaria será meritoria o de calidad, si se deriva de la búsqueda científica, y la investigación para cumplir con su misión académica y social, debe ser divulgada oportuna y sistemáticamente, mediante la interacción de los actores principales en los procesos de mediación del aprendizaje. En tal sentido, enseñar es una actividad rigurosa orientada, según García (2010: 66), "a construir ciudadanía (nacional y global) y no solamente enseñanza de competencias", implica crear las posibilidades de generar o construir conocimiento nuevo, para ello el profesor en su praxis debe promover la curiosidad indagadora.

En relación con esto último, las universidades deben cumplir con su responsabilidad social, de acuerdo con Vallaeys et al (2007), mediante la misión de la organización universitaria en los siguientes términos; en funcionamiento organizacional, educativo o formativo, cognoscitivo y epistemológico, así como en lo social, pues su responsabilidad debe ser a lo interno de la universidad, como al entorno o en su área de influencia. En ese sentido, los profesores deben cumplir con la misión de la universidad, a través de la práctica mediadora de la función docente, investigación, extensión y gestión institucional.

\section{La práctica mediadora en la función docente.}

La práctica mediadora en la función docente, la debe considerar el profesor como una actividad de la ciencia práctica o productiva, fundamentada el valor explicativo, lógico formal, según Castillo y Cabrerizo (2006: 7), "es un macrosistema de conocimientos que implica una organización de los contenidos conocidos y una organización de las formas de conocer esos contenidos". Así, el profesor organiza los contenidos existentes, los propósitos y genera mecanismos didácticos para el logro del hecho educativo, genera conocimiento y hace ciencia.

Por otra parte, la didáctica como disciplina, de acuerdo con Castillo y Cabrerizo (2006: 5), "integrante de las ciencias de la educación tiene como finalidad última el estudio del hombre, en cuanto ser educable y con capacidad para aprender"; su núcleo esencial es mediar en el aprendizaje, para que cada persona se haga autónoma, mediante el aprendizaje y las experiencias sistematizadas.

En tal sentido, la didáctica le permite al profesor mediar de manera asequible entre los estudiantes y los contenidos de las disciplinas que administra aun los más difíciles, según Doberti (2008: 124) "enseñar contiene entre sus dimensiones ineludibles la indicación de la concreta existencia de las realizaciones y la capacitación para la ejecución de las operaciones que la posibilitan"; mediante la producción de cambios conceptuales, al transformar al estudiante en un activo procesador de la información para darle sentido y construir conocimiento.

En otras palabras, la enseñanza es un acto didáctico que se vale de modelos, métodos y técnicas para promover aprendizajes significativos en los estudiantes mediante una adecuada interacción de estos con el profesor, así como con sus compañeros y el contexto. En este sentido, el docente que posee competencias didácticas hace de la clase y de la relación con los estudiantes una situación de aprendizaje a través de la conjugación de teoría, práctica e investigación.

Por consiguiente, el profesor en su práctica mediadora en el marco de la responsabilidad social universitaria, de acuerdo con Vallaeys et al (2009), influye sobre la formación de los profesionales en su manera de entender e interpretar el mundo, comportarse en ély valorar ciertas cosas en su vida. Por ello, promueve la deontología, orienta la definición de la ética profesional de cada disciplina y su rol social, al acordar los objetivos de aprendizaje, activar los conocimientos previos, el trabajo en equipo, el uso de analogías, contextualización y problematización de los contenidos, la reflexión sobre la práctica y la sistematización de las experiencias, lo que genera conocimiento y productividad investigativa. 


\section{Función de investigación}

La investigación, en el marco de las funciones del profesor universitario es definida, por el Programa de Beneficio Académico de los Profesores Universitarios de Venezuela (2002), como:

... el proceso de búsqueda constante de la excelencia, mediante formas diversas de descubrimiento, generación de innovaciones y tecnologías, aplicación y divulgación de conocimientos, análisis crítico y expresiones artísticas diversas, las cuales abarcan un amplio espectro de procesos, actividades y productos que constituyen la expresión del talento humano en sus múltiples manifestaciones: científicas, artísticasy humanísticas, realizadas en forma individual como en grupo. (p. 34)

Con relación a lo expresado, en el proceso de búsqueda de la excelencia, el profesor universitario en su actividad docente hace uso de la función de investigación apoyado en las líneas de investigación, a través de proyectos individuales y/o colectivos, donde se integren los contenidos en función de las necesidades e intereses de los estudiantes y del entorno, con el propósito de mejorar la calidad de los aprendizajes; así como de la extensión para divulgar los conocimientos y contribuir a mejorar las condiciones de vida de la comunidad o del entorno, a través de la generación de proyectos de investigación.

En el mismo orden de ideas, es importante considerar que la docencia e investigación, según Doberti (2008: 131), "son dos tareas básicas y constitutivas de la universidad que se esperan y exigen a los profesores con mayor dedicación", lo que refleja la naturaleza de la universidad y la relación entre la producción de conocimiento y la mediación, donde los profesores a tiempo completo y dedicación exclusiva son los encargados de concretarla.

Es evidente entonces, que se hace investigación desde la docencia cuando el profesor orienta a los estudiantes, según Gómez (2008: 136), "a descubrir el problema, a abordarlo y a resolverlo, apelando sin excepción a argumentos críticamente aceptables, procede a adquirir conocimiento". Es decir, cuando logra determinar la existencia del problema, se formulan las interrogantes, indaga, confronta el contenido con la realidad y propone soluciones, de esta manera el estudiante aprende investigando.

Resulta oportuno, afirmar que la universidad en el marco de la responsabilidad social, de acuerdo con Vallaeys et al (2009), orienta la producción del saber y las tecnologías, influye en la definición de lo que se llama socialmente verdad, ciencia, enseñanza, entre otras categorías, Incentiva (o no) la fragmentación y separación de los saberes al participar en la delimitación de los ámbitos de cada especialidad. A su vez, articula la relación entre tecnociencia y sociedad, promueve la democratización de la ciencia e influye sobre la definición y selección de los problemas de la agenda científica, mediante el estudio de los problemas del contexto, los proyectos de investigación, la participación en congresos científicos, políticas de de publicación y aplicación del conocimiento.

\section{Función de extensión}

La extensión es una función universitaria, concretada en su quehacer por el profesor universitario, definida por el Programa de Beneficio Académico de los Profesores Universitarios de Venezuela (2002), como:

... la proyección, en forma integrada de las funciones de docencia e investigación, mediante la interacción permanente de la universidad con la comunidad local, regional o nacional, con el propósito de contribuir a generar, aplicar y difundir conocimientos científicos, tecnológicos, artísticos y humanísticos, así como fomentar y promover actividades culturales y deportivas y contribuir a la solución de los problemas comunitarios, a través de programas y modalidades que respondan a las necesidades y expectativas de las personas y grupos interesados. (p. 45).

Sobre la base de las consideraciones anteriores, la función de extensión le permite a los profesores proyectar el trabajo científico de la universidad hacia la comunidad, para lo cual se vale de la docencia y de la investigación para generar, integrar, aplicar y difundir conocimientos en todos los campos del saber, fomentar actividades culturales, deportivas; así como contribuir a la solución de problemas que afectan a la sociedad.

En ese sentido, la función de extensión es un proceso, según Morales (2002), dinámico y permanente que no se queda aislada, sino que debe tener un vínculo con la enseñanza y la investigación. Por intermedio de ella, la universidad presta sus servicios a la sociedad, principalmente en las comunidades más carentes, mediante la mejora de recursos los cuales aumentan la calidad y expectativa de vida de los más necesitados.

Como puede observarse, la universidad en el marco de la responsabilidad social, de acuerdo con Vallaeys et al (2009), es un referente y un actor social, que puede promover (o no) el progreso, crear (o no) capital social, vincular (o no) la educación de los estudiantes con la realidad social exterior, entre otras. Es decir, la extensión universitaria, de acuerdo con Pérez (2007: 96) "es la función social de la universidad que exige un esfuerzo amplio de todos los actores que intervienen en el desarrollo de la misma, desde una visión integral e integradora de la docencia e investigación".

En efecto, el profesor en su praxis docente debe promover la interacción permanente de la universidad con el entorno, elaborar proyectos de asistencia a la comunidad, mediante la prestación de servicios profesionales educativos o asistenciales a organizaciones extrauniversitarias públicas o privadas, no gubernamentales, comunitarias, mediante convenios establecidos, puede ofrecer con sus estudiantes asesorías al sector educativo, participación en las actividades de la comunidad, prestación de servicios 
profesionales e intercambio de saberes de manera articulada con todas las partes orgánicas de la universidad.

\section{METODOLOGÍA}

La investigación se realizó en el marco del enfoque cuantitativo, el cual en opinión de Vieytes (2004: 33), tiene como objetivo "mostrar la realidad sin manipularla. Considerando a su vez que todo enunciado tiene sentido si es verificable". El tipo de investigación es analítica, pues el objetivo está dirigido a analizar la docencia en la educación superior como ciencia productiva en el marco de la responsabilidad social del profesor universitario en el Programa Educación de la Universidad Nacional Experimental Rafael María Baralt, de acuerdo con Hurtado (2010), el análisis es un procesamiento reflexivo, lógico, cognitivo el cual implica abstraer diversas pautas de relaciones internas que se ejecutan dentro de un evento, situación o fenómeno.

Con relación a lo descrito, los diseños de investigación analítica, según Méndez (2009), cumplen con los mismos criterios de las descriptivas y comparativas, donde se intentará a partir de un diagnóstico previo (estadio descriptivo) analizar los elementos que intervienen en una situación determinada. Por tanto, el estudio presenta un diseño de investigación no experimental, pues de acuerdo con lo expuesto por Hernández et al (2008: 205), es "la que se realiza sin manipular deliberadamente variables, lo que se hace es observar los fenómenos tal como se dan en su contexto natural, para después analizarlos". Es transeccional o transversal, por cuanto los datos de los profesores del Programa Educación de la UNERMB, se recolectaron en un único momento.

Con relación a la población del estudio, se constituyó por cuatrocientos diez (410) sujetos (profesores) distribuidos en las diferentes sedes del Programa Educación de la UNERMB (Cabimas, Ciudad Ojeda, Mene Grande y Los Puertos de Altagracia), del estado Zulia, para la elección de la muestra se recurrió a la fórmula propuesta por Sierra (2005: 227), aplicada en poblaciones finitas, en tal sentido se obtuvo una muestra de 80 sujetos, utilizándose el muestreo probabilístico de tipo estratificado, de acuerdo con Vieytes (2004), para la selección de los docentes por sedes.

Para la recolección de los datos se utilizó la técnica de la observación, según Tamayo y Tamayo (2008), es aquella donde el investigador puede observar y recoger datos mediante la encuesta. En ese sentido, se elaboró un instrumento tipo cuestionario de 69 itemes con escala frecuencial tipo Likert de cinco alternativas de respuesta: (5. Totalmente de acuerdo; 4. De acuerdo; 3. Indiferente; 2. En desacuerdo; 1. Totalmente en desacuerdo), que responden a las dimensiones e indicadores de medición de la variable del estudio.

El cuestionario fue sometido a validez por juicio de siete expertos y la confiabilidad mediante el método Alpha de Cronbach, obteniendo un valor de 0,894 ; considerado altamente confiable para su aplicación. Los datos se procesaron mediante la estadística inferencial, con la prueba Tukey y el Anova de un factor, utilizando el programa computarizado SPSS V. 19; se elaboró un baremo que permitió analizar, interpretar y confrontar los resultados con la teoría.

\section{ANÁLISIS Y DISCUSIÓN DE LOS RESULTADOS}

Con el propósito de analizar los resultados, se utilizó el programa SPSS Versión 19, para calcular los descriptivos de cada indicador que conforman las dimensiones: Práctica mediadora en la función: Docente, Investigación y Extensión y la aplicación del Anova, para determinar si existe diferencias significativa entre ellos. Así mismo, se diseño un baremo de interpretación de medias (Ver tabla $\mathrm{N}^{\circ} 1$ ) y los resultados obtenidos se muestran a través de tablas.

Tabla No 1. Baremo de interpretación de las medias

\begin{tabular}{|l|c|l|}
\hline \multicolumn{1}{|c|}{ Alternativas } & Rango & \multicolumn{1}{c|}{ Categoría } \\
\hline Totalmente de acuerdo & $4,21-5$ & Muy Alta \\
\hline De acuerdo & $3,41-4,2$ & Alta \\
\hline Indiferente & $2,61-3,4$ & Moderada \\
\hline En desacuerdo & $1,81-2,6$ & Baja \\
\hline Totalmente en desacuerdo & $1-1,8$ & Muy baja \\
\hline
\end{tabular}

Fuente: Elaboración propia

Previa aplicación del Anova, se realizó la prueba de Homogeneidad de las varianzas, para determinar si los grupos están en igualdad de condiciones (Ver tabla 2). Para ello se plantearon las siguientes hipótesis estadísticas:

$\mathrm{H}_{0}$ : las varianzas son iguales:

$\mathrm{H}_{1}$ : alguna de las varianzas es diferente

Si sig es menor que 0,05 se rechaza la hipótesis nula y se acepta la hipótesis alternativa.

Si sig es mayor que 0,05 , se acepta la hipótesis nula y se rechaza la hipótesis alternativa.

Tabla No 2. Prueba de homogeneidad de varianzas

\begin{tabular}{|c|c|c|c|}
\hline \multicolumn{4}{|c|}{ Ítemes } \\
\hline Estadístico de Levene & gl1 & gl2 & Sig. \\
\hline, 845 & 7 & 1912 &, 550 \\
\hline
\end{tabular}

Fuente: Datos aportado por SPSS V.19

En la tabla 2, se observa que el sig es mayor que 0,05 , por lo que se acepta que las varianzas son iguales, eso quiere decir que los grupos son homogéneos y se puede aplicar el Anova. 
Para determinar si entre los diferentes indicadores de las dimensiones, existe o no diferencia significativa entre ellos, se plantean las siguientes hipótesis estadísticas:

$\mathrm{H}_{0}: \mu_{1}=\mu_{2}=\mu_{3} \ldots . . \mu_{\mathrm{n}}$ no existe diferencia significativa entre los grupos

$\mathrm{H}_{1}: \mu_{1} \neq \mu_{2}$ existe diferencia significativa entre los grupos

Después de determinar que las varianzas son iguales, en la tabla $\mathrm{N}^{\circ} 3$, se muestra el resultado de la aplicación del Anova, en la cual se puede observar que el valor obtenido para inter grupos es menor de $\alpha=0,05$, se pudiera afirmar que si existe diferencia significativa, pero el valor de $\mathrm{F}=2,084$, es muy bajo y el $\operatorname{sig}=0,042$, está muy cerca de 0,05 , por lo que no se puede establecer que esa diferencia sea significativa. Pues si F (Prueba de Fisher) es mayor y menor el grado de significancia (Sig), es probable que exista diferencia significativa entre los grupos.

Tabla No 3. ANOVA de un factor

\begin{tabular}{|c|r|c|c|c|c|}
\hline \multicolumn{7}{|c|}{ ANOVA } \\
\hline & $\begin{array}{c}\text { Suma de } \\
\text { cuadrados }\end{array}$ & Gl & $\begin{array}{c}\text { Media } \\
\text { cuadrática }\end{array}$ & F & Sig. \\
\hline Inter-grupos & 11,958 & 7 & 1,708 & 2,084 &, 042 \\
\hline Intra-grupos & 1567,529 & 1912 &, 820 & & \\
\hline Total & 1579,487 & 1919 & & & \\
\hline
\end{tabular}

Fuente: SPSS V. 19

En la tabla 4, se observan los descriptivos para los indicadores de la práctica mediadora en la función docente, dando como resultado una media de 4.42; la cual según el baremo (Ver tabla 1) establecido, indica muy alta presencia de la práctica mediadora en la función docente del profesor universitario en el Programa Educación de la UNERMB; con un alto posicionamiento del uso de analogías, activación de conocimientos, trabajo en equipo, contextualización y problematización de los contenidos y reflexión sobre la praxis docente, mientras que la sistematización de experiencias ocupó un menor posicionamiento ante la docencia como ciencia productiva.

En la tabla $\mathrm{N}^{\circ} 5$, se presenta los resultados de la prueba de Tukey, en la cual se aprecian dos subconjuntos de medias homogéneas, donde los indicadores Uso de analogías y sistematización de experiencias son los dos indicadores que si presentan diferencias significativas.

Tabla No 5. Subconjuntos homogéneos

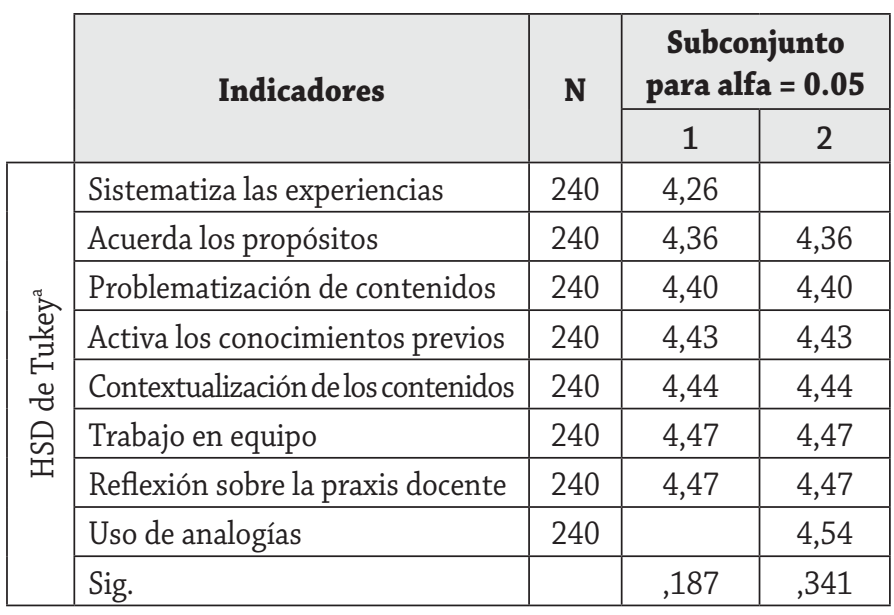

Se muestran las medias para los grupos en los subconjuntos homogéneos. ${ }^{\text {a }}$ Media armónica $=4,43$. Fuente: datos aportados por el SPSS V. 19.

Tabla No 4. Descriptivos de la dimensión: práctica mediadora en la función docente

\begin{tabular}{|c|c|c|c|c|c|c|c|c|}
\hline \multirow{2}{*}{ Indicadores } & \multirow{2}{*}{$\mathbf{N}$} & \multirow{2}{*}{ Media } & \multirow{2}{*}{$\begin{array}{l}\text { Desviación } \\
\text { típica }\end{array}$} & \multirow{2}{*}{$\begin{array}{l}\text { Error } \\
\text { típico }\end{array}$} & \multicolumn{2}{|c|}{$\begin{array}{c}\text { Intervalo de confianza para } \\
\text { la media al } 95 \%\end{array}$} & \multirow{2}{*}{ Mínimo } & \multirow{2}{*}{ Máximo } \\
\hline & & & & & $\begin{array}{l}\text { Límite } \\
\text { inferior }\end{array}$ & $\begin{array}{l}\text { Límite } \\
\text { superior }\end{array}$ & & \\
\hline Acuerda los propósitos & 240 & 4,36 & 979 &, 063 & 4,23 & 4,48 & 1 & 5 \\
\hline Activa los conocimientos previos & 240 & 4,43 & 912 & ,059 & 4,31 & 4,54 & 1 & 5 \\
\hline Trabajo en equipo & 240 & 4,47 & 882 & ,057 & 4,35 & 4,58 & 1 & 5 \\
\hline Uso de analogías & 240 & 4,54 & 862 & ,056 & 4,43 & 4,65 & 1 & 5 \\
\hline $\begin{array}{l}\text { Contextualización } \\
\text { de los contenidos }\end{array}$ & 240 & 4,44 & ,903 &, 058 & 4,32 & 4,55 & 1 & 5 \\
\hline Problematización de contenidos & 240 & 4,40 & 895 & ,058 & 4,29 & 4,51 & 1 & 5 \\
\hline Reflexión sobre la praxis docente & 240 & 4,47 & 905 & ,058 & 4,35 & 4,58 & 1 & 5 \\
\hline Sistematiza las experiencias & 240 & 4,26 & ,901 &, 058 & 4,14 & 4,37 & 1 & 5 \\
\hline Total & 1920 & 4,42 & ,907 & ,021 & 4,38 & 4,46 & 1 & 5 \\
\hline
\end{tabular}

Fuente: SPSS. V.19 
El resultado anteriormente descrito, coincide con el planteamiento de Castillo y Cabrerizo (2006: 7), pues "los conocimientos requieren una cierta estructuración y jerarquización de los mismos: algunos conocimientos deben ser principios: premisas, hipótesis, interpretaciones, axiomas, causas, leyes, etc." implica que el profesor en la actividad mediadora debe utilizar la organización de los conocimientos mediante la sistematización de la experiencias para tener carácter científico.

Seguidamente se analizó la Dimensión: Función investigación, conformada por los siguientes indicadores: problemas del contexto, proyectos de investigación, eventos científicos, publicaciones y aplicación del conocimiento obtenido en investigaciones, a través de la aplicación del Anova de un factor (Ver Tabla N 6). Como se puede observar el sig < 0,05, se rechaza la hipótesis nula, eso quiere decir que existen diferencias significativas entre los indicadores que conforman los diferentes grupos.

Tabla No 6. Anova de un factor

\begin{tabular}{|l|r|c|r|r|r|}
\hline & $\begin{array}{c}\text { Suma de } \\
\text { cuadrados }\end{array}$ & gl & $\begin{array}{r}\text { Media } \\
\text { cuadrática }\end{array}$ & F & Sig. \\
\hline Inter-grupos & 57,567 & 4 & 14,392 & 18,210 &, 000 \\
\hline Intra-grupos & 944,412 & 1195 &, 790 & & \\
\hline Total & 1001,979 & 1199 & & & \\
\hline
\end{tabular}

Fuente: SPSS. V.19

En la tabla $\mathrm{N}^{\circ} 7$, se presenta el análisis descriptivo de los indicadores que conforman esa dimensión, además de la media de cada indicador muestra la media y la deviación típica total de la dimensión. En la misma se observa que la media total es 4,43 , que según el Baremo establecido indica muy alta presencia de la función investigación en el profesor universitario en el Programa Educación de la UNERMB. El indicador proyectos de investigación obtuvo una media de 4,0375 ubicado en alta presencia según el Baremo, es el que está menor posicionado de todos los indicadores.

De acuerdo con los resultados expuestos, se puede inferir que los profesores creen que es necesario realizar investigaciones financiadas por organismos de la universidad, que los proyectos de investigación se deben desarrollar en redes interinstitucionales y están de acuerdo que las investigaciones se realicen con la participación activa de los estudiantes.

En la tabla No 8, se visualiza la prueba de TuKey, en ella se observan tres subconjuntos homogéneos de medias, donde los proyectos de investigación, problemas del contexto y los congresos científicos son los que estadísticamente más difieren significativamente.

Tabla No 8. Subconjuntos homogéneos

\begin{tabular}{|c|c|c|c|c|c|}
\hline & \multirow[t]{2}{*}{ dimens2 } & \multirow[t]{2}{*}{$\mathbf{N}$} & \multicolumn{3}{|c|}{$\begin{array}{c}\text { Subconjunto para } \\
\text { alfa }=0.05\end{array}$} \\
\hline & & & 1 & 2 & 3 \\
\hline \multirow{6}{*}{ 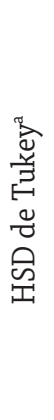 } & $\begin{array}{l}\text { proyectos de } \\
\text { investigación }\end{array}$ & 240 & 4,0375 & & \\
\hline & problemas del contexto & 240 & & 4,3583 & \\
\hline & $\begin{array}{l}\text { aplicación del } \\
\text { conocimiento obtenido } \\
\text { en investigaciones }\end{array}$ & 240 & & 4,5167 & 4,5167 \\
\hline & Publicaciones & 240 & & 4,5750 & 4,5750 \\
\hline & congresos científicos & 240 & & & 4,6583 \\
\hline & Sig. & & 1,000 & 059 & ,406 \\
\hline
\end{tabular}

Se muestran las medias para los grupos en los subconjuntos homogéneos. a Media armónica = 4,43. Fuente: datos aportados por el SPSS V. 19 .

Como se puede observar en la tabla 8, el indicador participación en congresos científicos obtuvo una media de 4,65, ubicado en la categoría muy alta, ocupando el mejor posicionamiento, pues los

Tabla N ${ }^{0}$ 7. Descriptivos de la dimensión: práctica mediadora en la función investigación

\begin{tabular}{|c|c|c|c|c|c|c|c|c|}
\hline \multirow{2}{*}{ Indicadores } & \multirow{2}{*}{$\mathbf{N}$} & \multirow{2}{*}{ Media } & \multirow{2}{*}{$\begin{array}{l}\text { Desviación } \\
\text { típica }\end{array}$} & \multirow{2}{*}{$\begin{array}{l}\text { Error } \\
\text { típico }\end{array}$} & \multicolumn{2}{|c|}{$\begin{array}{c}\text { Intervalo de confianza para } \\
\text { la media al } 95 \%\end{array}$} & \multirow{2}{*}{ Mínimo } & \multirow{2}{*}{ Máximo } \\
\hline & & & & & $\begin{array}{l}\text { Límite } \\
\text { inferior }\end{array}$ & $\begin{array}{l}\text { Límite } \\
\text { superior }\end{array}$ & & \\
\hline Problemas del contexto & 240 & 4,36 & ,875 &, 057 & 4,25 & 4,47 & 1 & 5 \\
\hline proyect de invest & 240 & 4,04 & 1,114 & ,072 & 3,90 & 4,18 & 1 & 5 \\
\hline Congresos científicos & 240 & 4,66 & ,760 & 049 & 4,56 & 4,75 & 1 & 5 \\
\hline Publicaciones & 240 & 4,58 & 850 &, 055 & 4,47 & 4,68 & 1 & 5 \\
\hline Aplic del conoc & 240 & 4,52 & ,803 & ,052 & 4,41 & 4,62 & 1 & 5 \\
\hline Total & 1200 & 4,43 & 914 & 026 & 4,38 & 4,48 & 1 & 5 \\
\hline
\end{tabular}

Fuente: SPSS. V.19 
profesores encuestados están totalmente de acuerdo con participar en congresos científicos con ponencias sobre los resultados de las investigaciones, favoreciendo el intercambio de ideas en el área de desempeño, por ello, es importante que se organicen y participen en jornadas científicas para socializar los resultados de las investigaciones realizadas.

Los resultados anteriormente descritos, coinciden con Blanco (2007:34), quien refiere que los profesores universitarios deben "comunicar sus trabajos en eventos científicos, para dar a conocer, los avances orientados a transformar la realidad". En tal sentido, es importante resaltar que los profesores al establecer un dialogo con sus pares en eventos científicos, no sólo validan sus conocimientos sino que difunden y socializan el saber, contribuyendo con la formación del talento humano en la investigación.

Así mismo, Stenhouse (2002) y Doberti (2008), refieren que no hay enseñanza sin investigación, pues las aulas son laboratorios y los profesores deben ser los investigadores encargados de comprobar en ellas la teoría educativa para mejorar la enseñanza, a través de la indagación y promover en los estudiantes la actividad investigativa como vía para el aprendizaje.

Con relación a los resultados para la función de extensión, se analizaron a través de la aplicación del Anova de un factor, los indicadores: prestación de servicios profesionales, asesorías al sector educativo, intercambio de saberes y participación en actividades de la comunidad (Ver Tabla No 9).
Tabla No 9. ANOVA de un factor

\begin{tabular}{|l|r|c|r|c|c|}
\hline & $\begin{array}{c}\text { Suma de } \\
\text { cuadrados }\end{array}$ & gl & $\begin{array}{c}\text { Media } \\
\text { cuadrática }\end{array}$ & F & Sig. \\
\hline Inter-grupos & 5,278 & 3 & 1,759 & 2,664 &, 047 \\
\hline Intra-grupos & 631,296 & 956 &, 660 & & \\
\hline Total & 636,574 & 959 & & & \\
\hline
\end{tabular}

Fuente: SPSS. V.19

En la tabla 9, se observa que el sig= 0,047 menor que el $\alpha=0,05$, pero ese valor de sig es muy próxima a $\alpha$, por lo que podemos concluir que si existe alguna diferencia significativa, pero debe ser muy mínima.

Seguidamente en la tabla $N^{\circ} 10$, se visualiza la estadística descriptiva de los indicadores que conforman la función de extensión, con una media de 4,46; ubicada en la categoría muy alta, observándose que todos los indicadores están en esa misma categoría. En esta función los profesores están totalmente de acuerdo con desarrollar proyectos de trabajo que involucran a instituciones educativas extrauniversitarias, con la prestación de servicios como asesor en las actividades organizadas por la coordinación de servicio comunitario constituyendo de esta manera, un aporte al desarrollo de la comunidad.

Tabla $\mathbf{N}^{\circ}$ 10. Descriptivos de la dimensión: práctica mediadora en la función extensión

\begin{tabular}{|c|c|c|c|c|c|c|c|c|}
\hline \multirow{2}{*}{ Dimensión 3} & \multirow{2}{*}{$\mathbf{N}$} & \multirow{2}{*}{ Media } & \multirow{2}{*}{$\begin{array}{l}\text { Desviación } \\
\text { típica }\end{array}$} & \multirow{2}{*}{$\begin{array}{l}\text { Error } \\
\text { típico }\end{array}$} & \multicolumn{2}{|c|}{$\begin{array}{c}\text { Intervalo de confianza para } \\
\text { la media al } 95 \%\end{array}$} & \multirow{2}{*}{ Mínimo } & \multirow{2}{*}{ Máximo } \\
\hline & & & & & $\begin{array}{l}\text { Límite } \\
\text { inferior }\end{array}$ & $\begin{array}{l}\text { Límite } \\
\text { superior }\end{array}$ & & \\
\hline asesorías al sector educativo & 240 & 4,4083 & ,76531 & 04940 & 4,3110 & 4,5056 & 1,00 & 5,00 \\
\hline $\begin{array}{l}\text { participación en actividades de } \\
\text { la comunidad }\end{array}$ & 240 & 4,5625 & ,75174 &, 04852 & 4,4669 & 4,6581 & 1,00 & 5,00 \\
\hline prestación de servicios profesionales & 240 & 4,3750 & ,93793 &, 06054 & 4,2557 & 4,4943 & 1,00 & 5,00 \\
\hline intercambio de saberes & 240 & 4,5000 & ,78159 &, 05045 & 4,4006 & 4,5994 & 1,00 & 5,00 \\
\hline Total & 960 & 4,4615 & ,81473 & ,02630 & 4,4099 & 4,5131 & 1,00 & 5,00 \\
\hline
\end{tabular}

Fuente: SPSS. V.19

Así mismo en la tabla $\mathrm{N}^{\circ} 11$, se pueden visualizar los resultados de la prueba de Tukey, pudiéndose observar que todos los indicadores se agrupan en un solo subconjunto, interpretándose que todas las medias tienen similitudes de comportamiento. La participación en actividades de la comunidad se encuentra en el más alto posicionamiento y la prestación de servicios profesionales presenta un menor posicionamiento.
De acuerdo con los resultados anteriores, se puede señalar que en ese mismo nivel los profesores encuestados están totalmente de acuerdo con el intercambio de saberes de la universidad con su entorno, mediante actividades con la comunidad favorecen su vinculación social, que la sabiduría popular permite recircular el conocimiento científico y con la participación en proyectos de trabajo que involucran a organizaciones de la comunidad se favorece el aprendizaje. 
Tabla $\mathbf{N}^{\circ}$ 11. Subconjuntos homogéneos

\begin{tabular}{|c|c|c|c|c|}
\hline & \multirow{2}{*}{ Dimens3 } & \multirow[t]{2}{*}{$\mathbf{N}$} & \multicolumn{2}{|c|}{$\begin{array}{c}\text { Subconjunto para } \\
\text { alfa }=0.05\end{array}$} \\
\hline & & & 1 & 2 \\
\hline \multirow{5}{*}{ 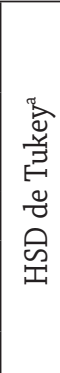 } & $\begin{array}{l}\text { prestación de servicios } \\
\text { profesionales }\end{array}$ & 240 & 4,3750 & \\
\hline & $\begin{array}{l}\text { asesorías al sector } \\
\text { educativo }\end{array}$ & 240 & 4,4083 & \\
\hline & intercambio de saberes & 240 & 4,5000 & \\
\hline & $\begin{array}{l}\text { participación en activi- } \\
\text { dades de la comunidad }\end{array}$ & 240 & 4,5625 & \\
\hline & Sig. & & ,056 & \\
\hline
\end{tabular}

Los resultados para el indicador participación en actividades de la comunidad, coinciden con Castillo y Cabrerizo (2006: 213), pues implica "incorporar las experiencias y que las reflexiones se hagan entorno a estas, que por ser más cercanas a la vida del profesor constituyen un aprendizaje significativo de tal forma que no se sientan ajenos a su contexto ni a su realidad cultural"; esta particularidad permite reforzar el ámbito cultural de las comunidades del área de influencia de la universidad donde el profesor ejerce la docencia.

Los hallazgos obtenidos para la función de extensión coinciden con Vallaeys (2005: 2), pues la responsabilidad social universitaria "nos exige preocuparnos por el mundo presente y futuro, ser actores partícipes de la solución de los más agudos problemas de nuestra sociedad". El profesor como ente corporativo, debe ser sensible ante los problemas de la sociedad en la cual desarrolla la actividad, por ello, la articulación de la docencia con la investigación y la extensión debe orientarse a resolver los problemas comunes, en función del bienestar social.

Tabla $\mathbf{N}^{\circ}$ 12. Comportamiento de la Variable: La docencia en educación superior como ciencia productiva en el marco de la responsabilidad social del profesor

\begin{tabular}{|l|c|c|c|}
\hline Medias & $\begin{array}{c}\text { Práctica } \\
\text { mediadora } \\
\text { en la función } \\
\text { docente }\end{array}$ & $\begin{array}{c}\text { Práctica } \\
\text { mediadora } \\
\text { en la función } \\
\text { investigación }\end{array}$ & $\begin{array}{c}\text { Práctica } \\
\text { mediadora } \\
\text { en la función } \\
\text { extensión }\end{array}$ \\
\hline $\begin{array}{l}\text { Media de la } \\
\text { dimensión }\end{array}$ & 4,42 & 4,43 & 4,46 \\
\hline $\begin{array}{l}\text { Media de la } \\
\text { Variable }\end{array}$ & \multicolumn{3}{|c|}{4,436} \\
\hline Categoría & \multicolumn{3}{|c}{ Muy Alta } \\
\hline
\end{tabular}

Fuente: Elaboración propia

En la tabla $\mathrm{N}^{\circ} 12$, se muestra el comportamiento de la variable La docencia en educación superior como ciencia productiva en el marco de la responsabilidad social del profesor universitario, se ubicó como muy alto dentro de las actividades de los docentes universitarios, ellos consideran que se puede hacer ciencia productiva a través de las funciones docencia e investigación y creen importante difundir ese conocimiento a través de la función extensión.

Los resultados descritos, coinciden con lo señalado por Pérez (2007: 96), quien afirma que "es la función social de la universidad que exige un esfuerzo amplio de todos los actores que intervienen en el desarrollo de la misma, desde una visión integral e integradora de la docencia e investigación"; por ello, como resultado de la actividad del flujo permanente de la universidad con el entorno, los profesores pueden elaborar proyectos de asistencia a la comunidad, mediante la prestación de servicios profesionales educativos o asistenciales a organizaciones extrauniversitarias, públicas o privadas, no gubernamentales o comunitarias, mediante convenios establecidos.

Es importante resaltar, que la formación profesional es pertinente cuando permita satisfacer las demandas de la sociedad y de la institución, en ese sentido enseñar en la educación universitaria no es transmitir información. Según García (2010: 66), "la educación sirve para construir ciudadanía (nacional y global) y no solamente enseñanza de competencias". Implica crear las posibilidades para que el alumno genere por si mismo su producción o su construcción de conocimiento nuevo, para ello el profesor en su praxis debe promover la curiosidad indagadora, convirtiéndose en una de las principales tareas de la práctica universitaria.

\section{CONCLUSIONES}

Sobre la base de los resultados obtenidos en la investigación, se presentan las siguientes conclusiones en las que se recogen las ideas centrales de cada uno de los hallazgos evidenciados de la confrontación de teorías, las cuales permitieron definir el comportamiento asumido por los docentes encuestados.

En relación con el objetivo dirigido a analizar la docencia en la educación superior como ciencia productiva en el marco de la responsabilidad social del Profesor universitario en el Programa Educación de la Universidad Nacional Experimental Rafael María Baralt, se concluye que existe muy alta presencia de la práctica mediadora en la función de docencia, investigación y extensión, considerando que los profesores en su trabajo académico son sensibles a los problemas de la sociedad en la cual se desarrolla la formación de profesionales.

En tal sentido, la docencia en educación superior como ciencia productiva en el marco de la responsabilidad social del profesor universitario, requiere del diseño de programas de trabajo basados en las necesidades de las comunidades, determinadas por motivos que originan la conducta de los actores y del tipo de relaciones que 
entre ellos se establezcan, con el propósito de asumir la responsabilidad al interior, satisfaciendo las necesidades de los trabajadores y al entorno, al participar en la gestión del conocimiento para resolver los problemas que afectan a los grupos sociales.

Cabe destacar, que en la práctica mediadora de la función docente, el uso de analogías obtuvo el mejor posicionamiento y la sistematización de experiencias de aprendizaje obtuvo el menor posicionamiento por lo que se hace necesario mejorar la rigurosidad en la docencia. En investigación, la participación en congresos científicos obtuvo mejor posicionamiento y los proyectos científicos el menor, por lo que es necesario promover la investigación para generar conocimiento y solucionar problemas. En extensión, obtuvo mejor posicionamiento la participación en actividades de la comunidad y el de menor posicionamiento fue prestación de servicios profesionales.

En efecto, los profesores en las actividades inherentes a las funciones de docencia, investigación y extensión, en el marco de la responsabilidad social universitaria, deben articular su trabajo en proyectos que surjan de las líneas de investigación existentes o que se puedan crear en las universidades a partir de las cátedras o departamentos, las cuales deben estar vinculadas con el contexto y la comunidad del entorno universitario, donde la docencia debe estar orientada a formar un profesional de calidad, que respete a la profesión como tal, consustanciado con las necesidades del entorno y que constituya equipos de trabajo, con autonomía y libertad académica.

\section{REFERENCIAS}

Albornoz Orlando (2010). Factores que influyen y condicionan la alta y baja tasa de productividad académica en América Latina y el Caribe. Informe de investigación financiada por el CENDES. Universidad Central de Venezuela. Caracas Venezuela.

Blanco, Carlos (2007). En resumen: discurso y conocimiento en la investigación educativa. Universidad Central de Venezuela. Consejo de Desarrollo Científico y Humanístico. Vicerrectorado Académico. Caras Venezuela.

Castillo Santiago y Cabrerizo Jesús. (2006). Formación del profesorado en educación superior. Didáctica y currículum. Volumen I. Editorial Mc Graw Hill. España.

Doberti R. (2008). Docencia e investigación: posiciones y oposiciones. En Pensar la Educación. Encuentros y desencuentros. Casalla María y Casalla Mario. Compiladores. Editorial Altamira. Argentina.

Fontaines Tomás. (2012). Metodología de la investigación. Pasos para realizar el proyecto de investigación. Júpiter editores C.A. Editorial Melvin. Caracas - Venezuela.

García Carmen. (2010). Educación superior comparada. El protagonismo de la internacionalización. Caracas Venezuela. IESALC - UNESCO / CENDES / bid \& co.editor. 2010. Colección Intramuros. Editorial Staff.
Gómez Ricardo. (2008). Educación y pensamiento crítico. En Pensar la Educación. Encuentros y desencuentros. Casalla María y Casalla Mario. Compiladores. Editorial Altamira. Argentina.

Hernández, Roberto; Fernández, Carlos; y Baptista, Pilar. (2008). Metodología de la investigación. Cuarta edición. Editorial Mc Graw Hill. México.

Hurtado, Jacqueline. (2010). Metodología de la investigación holística. Fundación Sypal. Ediciones Quirón. Caracas Venezuela.

Inciarte Alicia, Parra María, Bozo Ana. (2010). Reconceptualización de la Universidad. Una mirada desde América Latina. Ediciones Astrodata, S.A. Maracaibo. Venezuela.

Ley de Universidades (1970). Gaceta Oficial No 1429. Extraordinaria del 08-09-70. Congreso de la República de Venezuela. Caracas. Venezuela

Ley orgánica de ciencia, tecnología e innovación. (2005). Asamblea Nacional. Caracas Venezuela.

Méndez Carlos. (2009). Metodología. Diseño y desarrollo del proceso de investigación con énfasis en ciencias empresariales. Editorial Limusa. Cuarta edición. México.

Morales Laura. (2002). La extensión universitaria como proyección social. Universidad Pedagógica Experimental Libertador. Fedupel. Caracas.

Organización de las Naciones Unidas para la Educación, la Ciencia y la Cultura, UNESCO (2009). Declaración Final de la Conferencia Mundial sobre Educación Superior. La nueva dinámica de la educación superior y la investigación para el cambio social y el desarrollo. París. Francia. Recuperado de: http://www.unesco.org/education/WCHE2009/comunicado_es.pdf Consultado el 22/10/2012.

Pérez, Teresita. (2007). Caracterización de los vínculos de la extensión universitaria con las carreras de educación integral de la UNA. Un enfoque descriptivo, interpretativo e iluminativo. Fondo editorial Ipasme. Venezuela.

Programa del Beneficio Académico a los Profesores Universitarios de Venezuela (2002). Bases de la segunda convocatoria. Baremo. FAPUV-CNU. Caracas. Venezuela.

Ruz Yraima. (2009). Responsabilidad social de las instituciones públicas de educación superior en el Estado Zulia. Tesis Doctoral. Programa Doctorado en Ciencias de la Educación. Universidad Dr. Rafael Belloso Chacín. Maracaibo. Venezuela

Sierra Restituto. (2005). Tesis doctorales y trabajos de investigación científica. Editorial Paraninfo España.

Soto Felixberto. (2008). Impacto de la responsabilidad social universitaria en la conformación del perfil del egresado. Tesis Doctoral. Programa Doctorado en Ciencias de la Educación. Universidad Dr. Rafael Belloso Chacín. Maracaibo. Venezuela.

Stenhouse, Lawrence. (2002). Investigación y desarrollo del currículo. Editorial Morata. Madrid España.

Tamayo y Tamayo Mario. (2008) El Proceso de investigación Científica. Editorial Limusa. México.

Vallaeys, Françoise; De la Cruz Cristina, y Sasia Pedro. (2009). Responsabilidad Social Universitaria. Manual de primeros pasos. Mc Graw Hill. México. 
Vallaeys, Françoise; Carrizo Luis, y Oyasin Mitakuye (2008). ¿Compromiso social o RSU? Blog de ética y RSU. Reflexión crítica en torno a la ética aplicada a la RSU. En: http:// www.blog.pucp.edu.pe/item/23832/compromiso-social-oresponsabilidad-social-universitaria Consulta 22/10/2012.

Vallaeys Françoise; Carrizo Luis, y De La Cruz, Cristina. (2007). La responsabilidad social de las universidades. Pontificia Universidad Católica del Perú. En blog.pucp.edu.pe/item/21310k; consultado el 19/4/2009.
Vallaeys, Françoise. (2005). Hacia una conceptualización teórica de responsabilidad social universitaria. Pontificia Universidad Católica del Perú. Editorial Palestra. Perú.

Vera Luis, Gómez María, Acosta Yaneth y Perozo Lorheny. (2012). La docencia en el marco de la responsabilidad social universitaria. Revista Opción. Año 28.Nº 68. ISSN 1012-1587. Pp.257-272. Universidad del Zulia Maracaibo Venezuela.

Vieytes, Rut. (2004). Metodología de la Investigación en organizaciones, mercado y sociedad. Buenos Aires - Argentina: Editorial de las ciencias. 